\title{
IMPACTO FAMILIAR EN LA SOLUCIÓN DE PROBLEMAS QUE SE PRESENTAN COTIDIANAMENTE EN LA VIDA DE LOS NIÑNOS
}

Camila Londoño Galvis*, Karen Liseth León Buriticá**, David Gutiérrez ${ }^{* * *}$

\section{Resumen}

Este proyecto de investigación se basa en la construcción de conocimiento a partir de la influencia que tiene la familia en el desarrollo de los niños, teniendo en cuenta las formas de acompañamiento en la resolución de problemas y tareas que se presentan en su vida cotidiana y, a su vez, aprendiendo del contexto en el que se desenvuelven. En este caso se estudiarán las zonas rurales y citadinas en familias con niños entre 6 y 11 años de edad para conocer las diferentes formas de aprendizaje, participación y socialización tanto de la zona potencial como la zona real que interviene en el desarrollo del menor.

* Estudiante de Psicología, IV semestre, Fundación Universitaria del Área Andina. Correo: clondono42@estudiantes.areandina.edu.co

** Estudiante de Psicología, IV semestre, Fundación Universitaria del Área Andina. Correo: kleon13@estudiantes.areandina.edu.co

*** Asesor, docente, Fundación Universitaria del Área Andina, Grupo de investigación PAIDEIA. Correo: 


\section{Introducción}

Con el presente artículo, se quiere reconocer la influencia de la familia en la resolución de los diferentes problemas que van surgiendo constantemente en la vida de los niños y la participación que tienen los padres o cuidadores para ayudarles a completar diferentes actividades, y la importancia que tiene la familia en cualquier desarrollo. Por otro lado, Vygotsky habla de la zona de desarrollo próximo y la integración del desarrollo real y potencial; se toma también a Newman, Griffin y Cole, quienes explican la zona de construcción de conocimiento y expresan la importancia de la interacción interpersonal para el cambio cognitivo $y$, por último, basado en las formas de participación se tiene a Bárbara Rogoff, quien desarrolla la importancia de los roles decididos en un grupo de aprendizaje y las técnicas encontradas en estas actividades. En este caso, el contexto cultural es inherente, por lo que se realizará un comparativo entre las formas de enseñanza y aprendizaje en zonas rurales y citadinas.

Para la resolución de esta investigación se comenzará explicando la importancia de la influencia que puede tener la familia en la solución de los problemas de la vida cotidiana de los niños; por tanto, se trae a colación a Laura Isaza, quien afirma que el entorno familiar es el primer contexto donde el niño y la niña construyen sus aprendizajes,

el niño y la niña consiguen las cualidades primarias de subjetividad que lo diferencian como seres sociales y pertenecientes a un determinado régimen social. La familia es una unidad activa, flexible y creadora, es una institución que resiste y actúa cuando lo considera necesario, es una red de relaciones vividas. (Isaza, 2012, p. 1)

Ella afirma que las experiencias obtenidas en el entorno familiar son las que aportan habilidades sociales para la salida al mundo social.

De esta manera, se evidencia que las relaciones dentro de la familia son factores inherentes en el mundo social y educativo, lo cual da pautas para estar involucrado en ambos entornos; por consiguiente: "las relaciones interpersonales que se establecen entre los integrantes de la familia, que involucran aspectos de desarrollo, de comunicación, interacción y crecimiento personal, tienen una influencia directa en el desarrollo social de los hijos y las hijas" (Isaza, 2012). Así, la familia ayuda a construir las capacidades sociales y cognitivas del niño, lo cual ayuda a la toma de decisiones y a actuar según estas. Cortés y Cantón (2000) explican que la familia cumple un cargo importante en las funciones sociales de la vida del niño, por esto el clima familiar y las relaciones son inherentes para la explicación del nivel de adaptación.

Dentro de las actividades cotidianas presentadas en el clima familiar, se pueden encontrar problemas en los cuales la interacción del tutor y el niño son importantes para facilitar la culminación de este. Estas actividades o problemas que pueden surgir en cualquier momento son las que permiten un mayor aprendizaje en los menores, ya que no necesitan ser complejas para el desarrollo del cambio cognitivo del niño a par- 
tir del acompañamiento de sus tutores o cuidadores. Para aclarar esto se toma la explicación del cambio cognitivo que hace referencia a "la interacción dialéctica entre el mundo social y el cambio individual" (Newman et al., 1989). Esta dialéctica se compone de dos zonas que intervienen en este proceso: la primera, es la zona potencial, que hace referencia a la persona que guía, lidera y se encarga de exaltar las habilidades que el niño vaya mostrando; la segunda, se le denomina zona real a esas habilidades que el niño pueda emplear a partir de ellas o a partir de las cuales pueda realizar cualquier situación por sí solo. La interacción entre ambas zonas ayuda a darle un objetivo a la actividad de construcción del conocimiento.

A partir de las ideas que aportan estos autores, se tiene en cuenta que el desarollo individual del niño con el medio exterior es fundamental para la construcción de un aprendizaje, ya que es inherente la participación y socialización del pequeño con su mundo exterior. "Toda actividad, originada al interior de un colectivo, culminará por incidir sobre la construcción de las funciones superiores y algunos procesos solo pueden ser generados en actividades y procesos de socialización específicos" (Ruiz Carrillo y Estrevel Rivera, 2010); de acuerdo con estos procesos originados dentro de este colectivo, se evidencia la utilización de herramientas adquiridas en el proceso del cambio cognitivo. Estas se visualizan en la apropiación planteada en este caso por Leontiev, él explica que este es un proceso en el cual el experto facilita las herramientas y guías culturales que ayudan a reconocer y potenciali- zar las habilidades del novato, logrando, con base en estas, llevar al niño a cumplir un objetivo esperado. Sin embargo, se debe tener en cuenta que el niño debe llegar a cumplir su meta por sí solo, lo que significa que la actuación del experto sirve como base o sustento para que el novato construya sus propias capacidades. De esta manera, Newman et al. (1989) se apoyan en esta teoría y dan sustento a las dos partes que intervienen en la construcción del conocimiento, al explicar que la zona potencial se asemeja al experto y la zona real al novato, ambos roles cumplen con el proceso de aprendizaje. Después de este proceso, el novato es capaz de utilizar lo aprendido en un entorno diferente y puede llegar a una solución con los conocimientos aprendidos mediante esta interacción.

En este proceso se evidencia una clara necesidad de roles para facilitar el desarrollo de la actividad. Esta investigación tomará el aporte de Rogoff (1997) en su texto "Los tres planos de la actividad sociocultural", donde asegura que el aprendizaje se ha utilizado para comprender la relación entre el experto y el novato; sin embargo, el aprendizaje es un proceso más complejo, el cual se basa en un pequeño colectivo en donde se evidencian diferentes roles que permiten la consecución de metas y objetivos planteados. De esta manera, se potencializa el desarrollo de las destrezas y capacidades dentro de la actividad para la zona de construcción del conocimiento. Con esta teoría se pretende diferenciar las formas de participación de los miembros de la familia y los niños en el momento de realizar la actividad, al tener en cuenta la escolarización y las zonas 
culturales en las que se encuentren los miembros de la familia.

Así, es necesario familiarizarse con sectores, rasgos culturales y educativos con los que cuenta el entorno familiar, para lograr distinguir los contextos culturales y considerar las prácticas escolares que su cultura le otorga:

se puede decir que el desarrollo consiste en la apropiación de objetos, saberes, normas e instrumentos elaborados por la cultura dentro de contextos de actividad conjunta socialmente definidos, por ejemplo, la familia, la escuela o el trabajo, lo que hace que la educación tenga un rol inherente con el desarrollo. (Baquero, 1996; Bruner, 1988; Wertsch, 1988, 1993)

Los cuidadores proporcionan las técnicas y aportes, estos influyen en la educación de sus hijos apoyados en los aprendizajes que hayan recopilado gracias a su experiencia propia. Por esto, Espitia y Montes (2009) explican que los integrantes de la familia son quienes deben brindar las herramientas para fortalecer el proceso de aprendizaje, para que sea más fácil el desarrollo de sus hijos en su mundo social. Cabe destacar que esta

actividad de enseñanza y aprendizaje no se limita a un ámbito escolar, sino a toda situación en la que se confronten dos formas de hacer o saber. Así, el desarrollo se posibilita en casi cualquier acción humana, lo que lo hace más dinámico y vital. (Ruiz Carrillo y Estrevel Rivera, 2010)

Siguiendo este orden de ideas, las actividades por más cotidianas que sean, ten- drán una interacción que facilita la construcción del conocimiento dinámico.

Tomando en cuenta la población objeto de estudio, se abordará el contexto rural y citadino; por ende, se tomarán en cuenta los rasgos, instrumentos y escolaridad de los familiares, "el saber está conformado simultáneamente por procesos de apropiación, construcción y por productos culturales que se manifiestan en las prácticas discursivas y objetivadas en el conocimiento" (Núñez, 2004).

En este orden de ideas, al tener en cuenta todos los puntos abordados, se considera necesario construir una hipótesis que abarque con gran profundidad los temas que se quieren tratar. Es de suma importancia conocer la incidencia de los familiares en la construcción de aprendizajes y conocimientos en los niños y las niñas, elaborando la siguiente tesis para esta investigación: Influencia familiar en la zona de construcción de conocimiento con base en las formas de participación entre tutores y niños, para la solución de problemas en situaciones de la vida cotidiana que se presentan entre sus 6 y 11 años de edad, en familias de zonas rurales $y$ de zonas urbanas o citadinas.

Rogoff, junto a sus tres planos de la actividad sociocultural, propone algunos enfoques: aprendizaje o apprenticeship, participación guiada y apropiación participativa. Cada uno de estos enfoques muestra diferentes posturas o ideales que son de gran utilidad para alcanzar un aprendizaje óptimo en sociedad.

El aprendizaje o apprenticeship, muestra la constante relación que debe existir 
entre las personas logrando destacar los roles que cada quien cumple dentro de la sociedad, al entender los aportes que la persona puede dar y recibir.

La metáfora se centra en el papel activo de los recién llegados y del resto de los miembros del grupo en la organización de actividades y apoyo para el desarrollo de la participación, así como en las prácticas y metas culturales/institucionales de las actividades a las que contribuyen. (Rogoff, 1997)

En la participación guiada se presenta un sistema y estructura de compromisos interpersonales en el grupo encargado de fomentar el aprendizaje, en el que cada quien tiene sus propias actividades, pero siempre con el objetivo de complementarse entre los integrantes del grupo con actividades coordinadas y dirigidas. Por lo que Rogoff (1997) explica que:

es el término que he aplicado al plano interpersonal del análisis sociocultural. Este término enfatiza la implicación mutua entre los individuos y los otros miembros de su grupo, que se comunican y coordinan su implicación, en tanto participan en una actividad colectiva socioculturalmente estructurada.

Por otro lado, se encuentra la apropiación participativa, que es la transformación dada entre los individuos pertenecientes a un grupo por medio de diferentes procesos que permiten la participación para alcanzar un nivel de preparación eficaz, el cual los lleve a realizar actividades semejantes. Por ende, de acuerdo con Rogoff, es un "proceso por el cual los individuos transforman su comprensión de y su responsabilidad en el grupo a través de su propia participación. Esta noción está íntimamente ligada a las de aprendizaje y participación guiada” (Rogoff, 1997).

Sustentado en la zona de desarrollo próximo (ZDP), nombrado por Vygotsky, se elabora la importancia de este proceso para el cambio cognitivo mencionado por Newman, Giffin y Cole. La ZDP Vygotsky la definía como:

no es otra cosa que la distancia entre el nivel real de desarrollo, determinado por la capacidad de resolver independientemente un problema, y el nivel de desarrollo potencial, determinado a través de la resolución de un problema bajo la guía de un adulto o en colaboración con otro compañero más capaz. (Vygotsky, 1979, p. 133)

De esta manera, se crea un conocimiento que se utilizará en otros contextos $y$ en momentos diferentes como resultado de un aprendizaje. Vygotsky explicaba que las actividades que hoy se hacen con un acompañamiento, en un futuro se van a poder realizar de forma automática y autónoma, esto es posible gracias a la interacción entre el aprendizaje y el desarrollo propuesto en la zona de desarrollo próximo (Vygotsky, 1979).

A partir del proceso en la ZDP, se crea una interiorización, gracias a los conocimientos producidos por la interacción del medio social e individual. Esta interiorización es mediada por la cultura convirtiéndose después en una nueva función propia del sujeto,

[...] una operación que inicialmente 
construye y comienza a suceder internamente [...] un proceso interpersonal queda transformado en otro intrapersonal. En el desarrollo cultural del niño, toda función aparece dos veces: primero, a nivel social, y más tarde, a nivel individual. (Vygotsky, 1979).

Después de los procesos mencionados, se crean los procesos psicológicos superiores, que representan actividades cognitivas estructuradas del ser humano; estos se originan por las interacciones de los procesos nombrados anteriormente.

Basado en los aportes de Newman, Griffin y Cole en su libro La zona de construcción del conocimiento, se acoge la construcción de un cambio cognitivo a partir de las interacciones sociales.

Comenzando con la integración de las personas en diferentes grupos sociales, es necesario retomar las representaciones internas; los diferentes tipos de análisis y perspectivas son necesarios para el cambio cognitivo. Estas representaciones y estructuras cognitivas se ven reflejadas en la conducta del individuo; estas se observan en el momento de resolver la tarea. La postura que presentan los autores se basa en los cambios que se pueden producir en el sistema de métodos que se dan dentro del grupo y los mecanismos que se presentan en la actividad, que toman parte fundamental en este sistema y que sirven para el cambio cognitivo.

Un punto fundamental que debe tenerse en cuenta al momento de abarcar la zona de construcción de conocimiento es la direccionalidad, esto "hace referencia a los objetivos o metas de los sistemas de aprendizaje" (Newman et al., 1989), ex- plicando que si el niño repite la actividad después de un tiempo en situaciones diferentes, se entiende que el niño pasó a una siguiente etapa de conocimiento, apropiándose del tema en un factor cultural y contexto distinto. "Los niños descubren el objetivo de la tarea al participar en la interacción" (Newman et al., 1989).

\section{Objetivos}

- Comprender la manera en que influye el entorno familiar en la zona de construcción del conocimiento; así se reconoce la forma de acompañamiento en la resolución de problemas o eventos que se puedan presentar cotidianamente en la vida de los niños que se encuentran en las edades de 6 a 11 años en las zonas rurales y citadinas.

- Determinar el desarrollo real del niño; este se basa en las capacidades y habilidades que tiene el niño para solucionar problemas sin apoyo alguno, para seguir la línea de aprendizaje en la zona de construcción del conocimiento.

- Identificar las formas de acompañamiento que presentan los integrantes de la familia con el niño, visualizando el fortalecimiento de esta zona con la ayuda que puede prestar este para la solución de problemas.

- Conocer las formas de participación que tienen los niños con los cuidadores en el momento de solucionar la actividad que se presente.

- Analizar las diferencias en las formas de aprendizaje y enseñanza dilucidadas en familias de zonas rurales y citadinas, teniendo en cuenta que los implicados principales esta- 
rán en el rango de edad de los 6 a los 11 años y sus cuidadores principales con los que viven los niños.

\section{Metodología}

La presente investigación es de tipo cualitativo, la cual se caracteriza por ser descriptiva y basarse en la recolección de datos cualitativos, experiencias personales e historias de vida (Sampieri et al., 2014), que luego pasarán a ser interpretados y analizados a partir de la cultura en que se encuentren inmersos los participantes, en este caso zonas rurales y citadinas.

Al momento de realizar este tipo de investigación, lo ideal es tener un acercamiento inicial para conocer la problemática y así decidir las estrategias que mejor convengan en las zonas y poblaciones elegidas, reforzando las ideas que se hayan contemplado como idea inicial, ya que permitirá ampliar horizontes no tenidos en cuenta con anterioridad. Este tipo de investigación le da importancia al contexto y a los actos del ser humano, valora la realidad de las personas con sus sentimientos y motivaciones.

A partir de lo que se quiere conocer con esta investigación, se plantea implementar un enfoque de estudio de casos, que se desarrolla en grupos de estudio pequeños para una mayor profundidad al momento de trabajar con ellos, ya que se observa la interacción social de los implicados, teniendo en cuenta las relaciones sociales y las experiencias de los sujetos de estudio (Jiménez y Comet, 2016). La estrategia principal que se ha considerado pertinente emplear en este estudio es la observación participante: "La observación participante es el proceso que faculta a los investigadores a aprender acerca de las actividades de las personas en estudio en el escenario natural a través de la observación y participando en sus actividades" (Dewalt y Dewalt, 2002). De esta manera, se busca familiarizar estrechamente con el grupo de estudio elegido teniendo en cuenta las edades de los niños.

Para llevar a cabo estas estrategias metodológicas se plantea una población explícita, por lo que se toma un rango de edades entre los 6 y 11 años de edad, con el fin de identificar la zona de construcción del conocimiento y la influencia familiar. Este rango de edades hace referencia a la segunda infancia; en esta etapa se generan los aprendizajes básicos, ya que se adentra a su entorno social y hay mayor necesidad de acompañamiento de los tutores, por lo que tomamos la idea de Mansilla quien afirma:

La segunda infancia (que va de 6 a 11), se caracteriza por su apertura al mundo externo y por la acelerada adquisición de habilidades para la interacción. En este período, los niños tienen un riesgo menor que en la primera infancia. (Mansilla, 2000)

Explica que en la primera etapa, los niños empiezan a percibir el mundo que los rodea y a adquirir sus primeros conocimientos a partir de la experimentación, mientras que, en la segunda, esos conocimientos se pueden aplicar para adquirir nuevos y son un poco más conscientes del mundo que los rodea. 


\section{Discusión}

En esta investigación se puede distinguir la categoría psicológica que es la zona de construcción del conocimiento que trata de abarcar los puntos necesarios para un cambio cognitivo, relacionando diferentes teorías como la zona de desarrollo, también nombrada en este proyecto; aunque se encuentra un déficit a la hora de distinguir las formas de participación que pueden existir en las actividades por realizar. Por este motivo, se expresa en esta investigación la importancia de visualizar los roles, como lo expresa Bárbara Rogoff, que se pueden presentar en la solución de problemas y expresar la inherencia de los grupos para el proceso de aprendizaje y no limitarse a los procesos entre más capaz y menos capaz; por el contrario, integrar la sociedad como base para la transformación.

Teniendo en cuenta la necesidad de reconocimiento de estos procesos, se realizó un comparativo entre las zonas rural y citadina; sin embargo, esta investigación se llevó a cabo también con el fin de dar participación a esos contextos que no tienen mayor protagonismo; la zona rural es de gran importancia, pero se ha estudiado de manera arbitraria y muy poco profunda, por lo que se enfocó en las diferencias que existen entre ambas zonas, mostrando las formas de aprendizaje y la adquisición de habilidades y conocimiento a partir de los niveles o la facilidad de escolarización.

Por estos motivos se realizaron los planes para evidenciar y retomar las categorías

y vacíos que se encuentran en la relación de teorías, fortaleciendo así la investiga- ción de la construcción de aprendizajes en actividades cotidianas, estructurando las competencias que se deben visualizar en las diferentes actividades.

Fundamentado en los objetivos planteados, se conoce la subcategoría de la zona de construcción del conocimiento; de esta manera, se determina el desarrollo real de los implicados. En este se visualiza las habilidades y capacidades que pueda manejar y dominar sin ayuda o guía de un superior. Esto se basa en la teoría de Lev Vygotsky, en la que se demuestra la importancia de delimitar el desarrolo real para así identificar las formas de acompañamiento. Esta se identifica principalmente con los cuidadores comunes, se evidencia la interacción entre los tutores y el niño en el momento de desarrollar la tarea, y aquí se identifican las formas de acompañamiento, las guías y pautas que pueda presentar el tutor para la solución de este; teniendo en cuenta las guías se reconocen las formas de participación que tiene el niño en el desarrollo de la tarea y de qué manera emplea los aprendizajes que ha adquirido para solucionar esta actividad. Teniendo en cuenta la ejecución de tareas entre niños y cuidadores, se debe tener claro que para obtener un aprendizaje se deben basar en la cultura que están inmersos y de qué forma se desenvuelven en ella. En este caso, se aplican los espacios como la zona rural y la zona citadina, entendiendo el análisis entre ambos sectores como base para el aprendizaje de los procesos que las familias llevan a partir de su contexto.

Tomando como base la información teórica que abarca el tema, es necesario 
plantear la importancia de este proyecto, lo cual podría facilitar estrategias de intervención según el contexto descrito. En este caso, el impacto que se quiere lograr va dirigido al componente de responsabilidad social y cultural, ya que el tema que se está abordando va enfocado al reconocimiento del comportamiento humano a partir de las formas de educación y cultura en los diferentes espacios como la zona rural y la zona citadina, logrando analizar en ambos lugares las formas de acompañamiento, enseñanza $\mathrm{y}$ aprendizaje entre niños y cuidadores.

\section{Referencias}

1. Carrillo, E., Estrevel Rivera, L. B., \& Estrevel Rivera, L. B. (2010). Vygotsky: la escuela y la subjetividad. Pensamiento Psicológico, 8(15).

2. Cortés, R., \& Cantón, J. (2000). Ambiente familiar y dificultades de adaptación de los hijos. Suma Psicológica, 7(2), 33-49.

3. Dewalt, K., \& Dewalt, B. R. (2002). La observación participante: una guía para los trabajadores de campo. Walnut Creek, Estados Unidos.

4. Espitia, R., \& Montes, M. (2009). Influencia de la familia en el proceso educativo de los menores del barrio Costa Azul de Sincelejo (Colombia). Artículos de Investigación, 17(1), 84-105.
5. Isaza, L. (2012). El contexto familiar: un factor determinante en el desarrollo social de los niños y las niñas. Medellín, Colombia. Revista Electrónica de Psicología Social "Poiésis".

6. Jiménez, V., \& Comet, C. (2016). Los estudios de casos como enfoque metodológico. Académico. Revista de Investigación en Ciencias Sociales y Humanas, 3(2), 11.

7. Mansilla, M. (2000). Etapas del desarrollo humano. Revista de Investigación en Psicología, 3(2), 105-116.

8. Newman, D., Griffin, P., \& Cole, M. (1989). La zona de construcción del conocimiento: por un cambio cognitivo en la educación. Madrid (Ministerio de Educación y Ciencia).

9. Núñez, J. (2004). Los saberes campesinos: implicaciones para una educación rural. Investigación y Posgrado, 19(2), 44.

10. Rogoff, B. (1997). Los tres planos de la actividad sociocultural: "apropiación participativa", "participación guiada" y "aprendizaje”. En Wertsch, J., del Río, P. y Álvarez, A. (eds.). La mente sociocultural. Aproximaciones teóricas y aplicadas (pp. 111-128). Madrid: Fundación Infancia y Aprendizaje.

11. Sampieri, R., Fernández, C., \& Baptista, P. (2014). Metodología de la investigación. Sexta edición. México.

12. Vygotski, L. S. (1979). El desarrollo de los procesos psicológicos superiores. Barcelona. 